\title{
cmaJOPEN
}

\section{Adoption, feasibility and safety of a family medicine-led remote monitoring program for patients with COVID-19: a descriptive study}

\author{
Payal Agarwal MD, Geetha Mukerji MD MSc, Celia Laur PhD, Shivani Chandra MSc, Nick Pimlott MD, \\ Ruth Heisey MD, Rebecca Stovel MD MScCH, Elaine Goulbourne MSc, R. Sacha Bhatia MD MBA, \\ Onil Bhattacharyya MD PhD, Danielle Martin MD MPP
}

\section{Abstract}

Background: Virtual care for patients with coronavirus disease 2019 (COVID-19) allows providers to monitor COVID-19-positive patients with variable trajectories while reducing the risk of transmission to others and ensuring health care capacity in acute care facilities. The objective of this descriptive analysis was to assess the initial adoption, feasibility and safety of a family medicine-led remote monitoring program, COVIDCare@Home, to manage the care of patients with COVID-19 in the community.

Methods: COVIDCare@Home is a multifaceted, interprofessional team-based remote monitoring program developed at an ambulatory academic centre, the Women's College Hospital in Toronto. A descriptive analysis of the first cohort of patients admitted from Apr. 8 to May 11, 2020, was conducted. Lessons from the implementation of the program are described, focusing on measure of adoption (number of visits per patient total, with a physician or with a nurse; length of follow-up), feasibility (received an oximeter or thermometer; consultation with general internal medicine, social work or mental health, pharmacy or acute ambulatory care unit) and safety (hospitalizations, mortality and emergency department visits).

Results: The COVIDCare@ Home program cared for a first cohort of 97 patients (median age 41 yr, $67 \%$ female) with 415 recorded virtual visits. Patients had a median time from positive testing for severe acute respiratory syndrome coronavirus 2 (SARS-CoV-2) to first appointment of 3 (interquartile range [IQR] 2-4) days, with a median virtual follow-up time of 8 (IQR 5-10) days. A total of 4 (4\%) had an emergency department visit, with no patients requiring hospitalization and no deaths; 16 (16\%) of patients required support with mental and social health needs.

Interpretation: A family medicine-led, team-based remote monitoring program can safely manage the care of outpatients diagnosed with COVID-19. Virtual care approaches, particularly those that support patients with more complex health and social needs, may be an important part of ongoing health system efforts to manage subsequent waves of COVID-19 and other diseases.

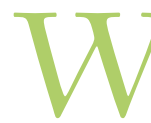

ith the global spread of the coronavirus disease 2019 (COVID-19) pandemic, Canada had 460743 cases and 13431 deaths as of Dec. 13, 2020. ${ }^{1}$ The initial health system response in Ontario was to reduce inperson care substantially, switching to virtual visits for most care. For patients with COVID-19, using virtual care to monitor the disease at home allows providers to address patient needs while reducing the risk of transmission to other patients or providers. ${ }^{2-4}$ Most patients who have tested positive for severe acute respiratory syndrome coronavirus 2 (SARSCoV-2) can safely convalesce at home, but about $10 \%$ will require hospital admission..$^{5-7}$ Importantly, some presentations require early identification and acute care treatment to prevent poor outcomes, ${ }^{8-10}$ whereas frequent virtual touch points may reduce unnecessary emergency department visits for others. ${ }^{2,6,11}$

Management of milder COVID-19 cases would ideally have been provided by a patient's own primary care provider, but many primary care providers in Ontario reduced services during the first wave of the pandemic as they adjusted to virtual care. In addition, the confidence of primary care providers in their ability to manage a novel infectious disease at a distance was often unclear. ${ }^{2,12}$ Furthermore, there is no consensus on the optimal model of remote monitoring for COVID-19. Some models are specialty based, ${ }^{6}$ and others include both primary care and specialty care physicians. ${ }^{5,13,14}$ These resource-intensive models may not be sustainable

\section{Competing interests: None declared.}

This article has been peer reviewed.

Correspondence to: Payal Agarwal, payal.agarwal@wchospital.ca CMAJ Open 2021. DOI:10.9778/cmajo.20200174 
with rising case counts and may not be generalizable to other contexts. Many models are disease focused and algorithm dependent, and are not designed to manage patient comorbidities and the inevitable psychosocial issues that arise during the illness. ${ }^{5,6,13,14}$

A family medicine-led interprofessional model of remote home monitoring for patients with COVID-19, COVIDCare@Home, was developed in an ambulatory academic centre in Toronto, with a focus on patients who did not have a pre-existing, close connection to primary care. In this study, we aimed to describe the model of care in COVIDCare@ Home and discuss its initial adoption, feasibility and safety in the first 5 weeks.

\section{Methods}

\section{Design and setting}

Women's College Hospital is an ambulatory academic hospital located in Toronto. In late March 2020, the hospital partnered with the Department of Family and Community Medicine at the University of Toronto, and Mount Sinai Hospital, an acute care academic hospital that is part of the Sinai Health System, to develop a model of care for patients with COVID-19 in the community. The program was operational on Apr. 8, 2020, with ongoing improvement cycles to improve the model of care delivery as the pandemic evolved. This article includes a descriptive analysis of all patients who had their first appointment from the start of the program until May 11, 2020.

\section{Care model}

COVIDCare@Home offers remote monitoring, using telephone or video visits, 7 days a week by an interprofessional, family medicine-led team for community-based patients with COVID-19 (Appendix 1, available at www.cmajopen.ca/ content/9/2/E324/supp1/DC1). The aim of the program was to follow patients during the acute phase of the illness (typically 14 days from symptom onset) or until they were discharged to community-based care from their primary care provider. The team included a family physician, a family medicine resident, a registered nurse, a mental health or social worker, a nurse practitioner and a pharmacist. Specialists, including specialists in general internal medicine, respirology and psychiatry, were available for virtual consultations. Patients also had access to a 24-hour on-call service. All clinicians were recruited from Women's College Hospital and typically worked 1 day a week in the program.

The clinical protocol for remote monitoring, including risk assessment, risk stratification and management plans, were based on clinical expertise and available evidence 2,15 (Appendix 2, available at www.cmajopen.ca/content/9/2/E324/suppl/ DC1). Multiple clinicians, including 6 family physicians, an emergency medicine physician, 2 general internists, a respirologist, an infectious diseases specialist, a psychiatrist and 2 health program administrators, provided iterative input into the clinical assessment tool through virtual meetings and online feedback led by experts in human-centred design.
Initial assessments of all patients were done by the resident, under supervision of the staff physician. Patients could participate in video visits (using a cellphone, tablet or computer) or by telephone, with access to translation services. Patients were triaged to low, moderate or high risk using clinical judgment. High-risk patients were identified using factors including the following: older than 60 years, presence of multiple comorbidities, trajectory in disease course,${ }^{16}$ clinical symptom of worsening shortness of breath or oxygen saturation less than $94 \%,{ }^{2}$ and additional social complexities. Follow-up virtual visits were booked with the resident or registered nurse every 1-3 days on the basis of risk. The team held daily huddles and weekly rounds with the full team and specialists to discuss challenging cases or common questions.

Pulse oximeters and thermometers, designed for home consumer use, were couriered to patients thought to be at high risk for respiratory decompensation (on the basis of older age; comorbid illness, including asthma, chronic obstructive pulmonary disease, diabetes and hypertension; and current respiratory symptoms). The nurse practitioner supported case management of patients with complex medical or mental health conditions, and social workers and mental health workers provided brief counselling and access to community resources. The team reached out to each patient's primary care provider (where relevant) to facilitate shared care and send a discharge plan. Care was charted using the Epic electronic medical record (EMR) at Women's College Hospital, which enables secure, EMR-integrated video visits via Zoom and bidirectional asynchronous messaging using a patient portal. A website with resources for patients and physicians was developed to facilitate care (COVIDcare@home.ca). A dashboard cataloguing each patient in the program with their risk level for deterioration and active care issues was developed to facilitate daily team huddles.

\section{Participants}

All community-dwelling patients in the Greater Toronto Area diagnosed with COVID-19 (positive test for SARS$\mathrm{CoV}-2$ or probable case based on the definition by the Ontario Ministry of $\mathrm{Health}^{17}$ ) were eligible for the program. The primary referral source was the COVID-19 assessment centre at Women's College Hospital. Once they received the results of a positive diagnosis, all patients were offered referral to the COVIDCare@Home program. Patients were also referred, on the basis of clinician judgment, from the Mount Sinai Hospital assessment centres and emergency department, and from acute care or in-patient rehabilitation services at Sinai Health System. Referred patients were excluded only if they did not have access to a phone. All consecutive patients who had their first visit during the study period were included in this study.

\section{Data sources}

Clinical and contextual information regarding patients and COVID-19 diagnosis was collected during virtual clinical encounters and entered into the Epic EMR using a standardized electronic flowsheet (Appendix 2). All data from the flow 
sheet on demographic characteristics and program utilization data were electronically extracted. Two researchers, who each received training from the study lead (P.A.) using 10 randomly selected charts, conducted the chart review. Each participant chart was reviewed by 1 researcher to verify data extracted from the flow sheet and to extract additional clinical information. Any identified data discrepancies were reviewed by the study lead (P.A.), and consensus was reached as a group. The study lead (P.A.) reviewed 10 randomly selected charts at the end of the data extraction to ensure accuracy of the data.

\section{Outcomes}

This study was designed to assess implementation outcomes including the adoption, feasibility and safety of the COVIDCare@Home program, as outlined by Proctor and colleagues for the assessment of early-stage innovation. ${ }^{18}$ Adoption, or "uptake," 18 was assessed by the number of patients referred who enrolled in the program, the average number of visits per patient, and the follow-up or virtual length of stay (days from first appointment to last appointment). Feasibility, or practicality of the program, ${ }^{18}$ was determined by assessing the utilization of resources and services, the access to the program (as measured by median time from positive swab to first appointment), the number of pulse oximeters and thermometers sent, and the number of consultations to social work, general internal medicine or other additional staff. Given the risk of acute morbidity and mortality for patients with COVID-19, safety of the program was also assessed using the rate of emergency department visits, hospitalizations and mortality among participants.

\section{Statistical analysis}

Descriptive analyses were used with continuous variables reported as medians and interquartile ranges (IQRs), and categorical variables reported as numbers and percentages.

\section{Ethics approval}

The study was approved by the local research ethics board at Women's College Hospital (2020-0058-E).

\section{Results}

Baseline demographic and clinical characteristics of patients in the COVIDCare@Home program are presented in Table 1. A total of 98 patients met the inclusion criteria, but 1 did not complete their initial appointment. The median age was 41 (IQR 31-58) years, and 65 (67\%) were female. Of 97 patients, $22(23 \%)$ did not have access to a primary care provider. Half $(n=49,51 \%)$ had at least 1 comorbidity, and 11 (11\%) had 3 or more comorbidities. Most patients $(88 \%)$ had positive swabs for SARS-CoV-2, but 5 patients $(5 \%)$ were unlikely to have COVID-19, and symptoms were later attributed to other causes.

More than half of participants $(n=55,57 \%)$ had an occupation that put them at high risk for SARS-CoV-2 infection. Of these, many patients were front-line health care workers,

\section{Table 1: Characteristics of patients enrolled in the} COVIDCare@Home program

\begin{tabular}{cc|}
\hline & No. $(\%)$ of patients \\
Characteristic & $n=97$
\end{tabular}

Age, yr

Median (IQR) $41(31-58)$

$<18 \quad 1(1)$

$>60 \quad 17(17)$

Sex, female $65(67)$

Had a primary care provider $\quad 75$ (77)

Comorbidities

Patients with $\geq 1$ comorbidities 49 (51)

Asthma $11(11)$

Autoimmune disorder or $6(6)$

immunosuppressed

$\begin{array}{ll}\text { Congestive heart failure } & 1(1) \\ \text { Chronic kidney disease } & 1(1)\end{array}$

Liver disease $0(0)$

COPD $1(1)$

Cardiovascular disease $2(2)$

Diabetes $6(6)$

Hypertension $11(11)$

Malignancy $0(0)$

Anxiety $9(9)$

Depression $3(3)$

Dyslipidemia $10(10)$

Other comorbidities $22(23)$

Other factors

Pregnancy

\begin{tabular}{|cc|}
\hline Positive SARS-CoV-2 test & $88(91)$ \\
\hline Probable case & $4(4)$ \\
\hline COVID-19 negative $\S$ & $5(5)$ \\
\hline Identified risk factors for acquiring COVID-19 & \\
\hline Occupation & $55(57)$ \\
\hline Long-term care home & $18(19)$ \\
\hline Acute care & $9(9)$ \\
\hline Shelter & $9(9)$ \\
\hline Complex continuing care & $12(12)$ \\
\hline Grocery store & $7(7)$ \\
\hline Travel & $6(6)$ \\
\hline
\end{tabular}

Note: $\mathrm{COPD}=$ chronic obstructive pulmonary disease, COVID-19 = coronavirus disease 2019, IQR = interquartile range, SARS-CoV-2 = severe acute respiratory syndrome coronavirus 2.

*Unless stated otherwise.

†Any current use of tobacco cigarettes.

$\ddagger$ Self-identified by the patient.

$\S$ Testing for SARS-CoV-2 was negative, and symptoms were later attributed to other causes. 
including 13 personal support workers (13\%), 11 nurses (11\%), 5 shelter workers (5\%), 1 physician (1\%), and 6 patients who worked in cleaning or environmental services in a health care setting $(6 \%)$.

\section{Adoption, feasibility and safety}

Table 2 highlights the adoption of the program. Over the study period, 97 patients had at least 1 visit, with a median of 4 (IQR 2-5) visits per patient; there was a total of 415 visits (62\% booked as video visits). The median virtual length of follow-up was 8 (IQR 5-10) days. Figure 1 includes details on

Table 2: COVIDCare@Home measures of program adoption

\begin{tabular}{|lc|}
\hline Measure & $\begin{array}{c}\text { No. (\%) or median (IQR) } \\
n=415 \text { visits }\end{array}$ \\
\hline $\begin{array}{l}\text { Visits with family physician } \\
\text { or resident }\end{array}$ & $251(60.5)$ \\
\hline Visits with registered nurse & $164(39.5)$ \\
\hline Visits per patient & $4(2-5)$ \\
\hline $\begin{array}{l}\text { Time from results of } \\
\text { SARS-CoV-2 testing to first } \\
\text { visit, } d^{*}\end{array}$ & $8(5-10)$ \\
\hline $\begin{array}{l}\text { Length of follow-up in } \\
\text { program, d } \dagger\end{array}$ & \\
\hline $\begin{array}{l}\text { Note: IQR }=\text { interquartile range, SARS-CoV-2 = severe acute respiratory } \\
\text { syndrome coronavirus 2. } \\
* n=94 ; 3 \text { patients were not tested for SARS-CoV-2. } \\
\text { tLength of follow-up is the time from the first appointment to the last. }\end{array}$ \\
\hline
\end{tabular}

the number of visits per patient, and Figure 2 shows the cumulative number of appointments by week. Table 3 documents program feasibility, including access and utilization of program services.

Program safety metrics, outlined in Table 4, show that 4 patients visited the emergency department while in the program, and 2 were referred through the program. There were no hospitalizations or deaths during the study period.

\section{Interpretation}

Analysis of the first 97-patient cohort in the COVIDCare@ Home program shows that a team-based, family medicine-led remote monitoring program for COVID-19 is feasible and safe. The median length of follow-up of 8 days in the program and the median of 4 visits per patient suggest strong patient adoption and retention over the typical time course of COVID-19. Preliminary analysis shows the program was safe, with limited need to transfer care to the emergency department, and no hospitalizations or deaths.

Remote monitoring programs have been described in the literature from several countries, including another from Canada (1 reference from preprint). 5,6,13,14,19,20 They vary in intensity of resource use, with COVIDCare@Home sitting between the low-intensity models that use low-touch monitoring, such as automated daily surveys, and those of higher intensity with multiple check-ins per day (1 reference from preprint). ${ }^{14,19}$ For programs with similar patient populations to COVIDCare@Home, the emergency department and hospital admission rates were all relatively low (1\%-12\%); however,

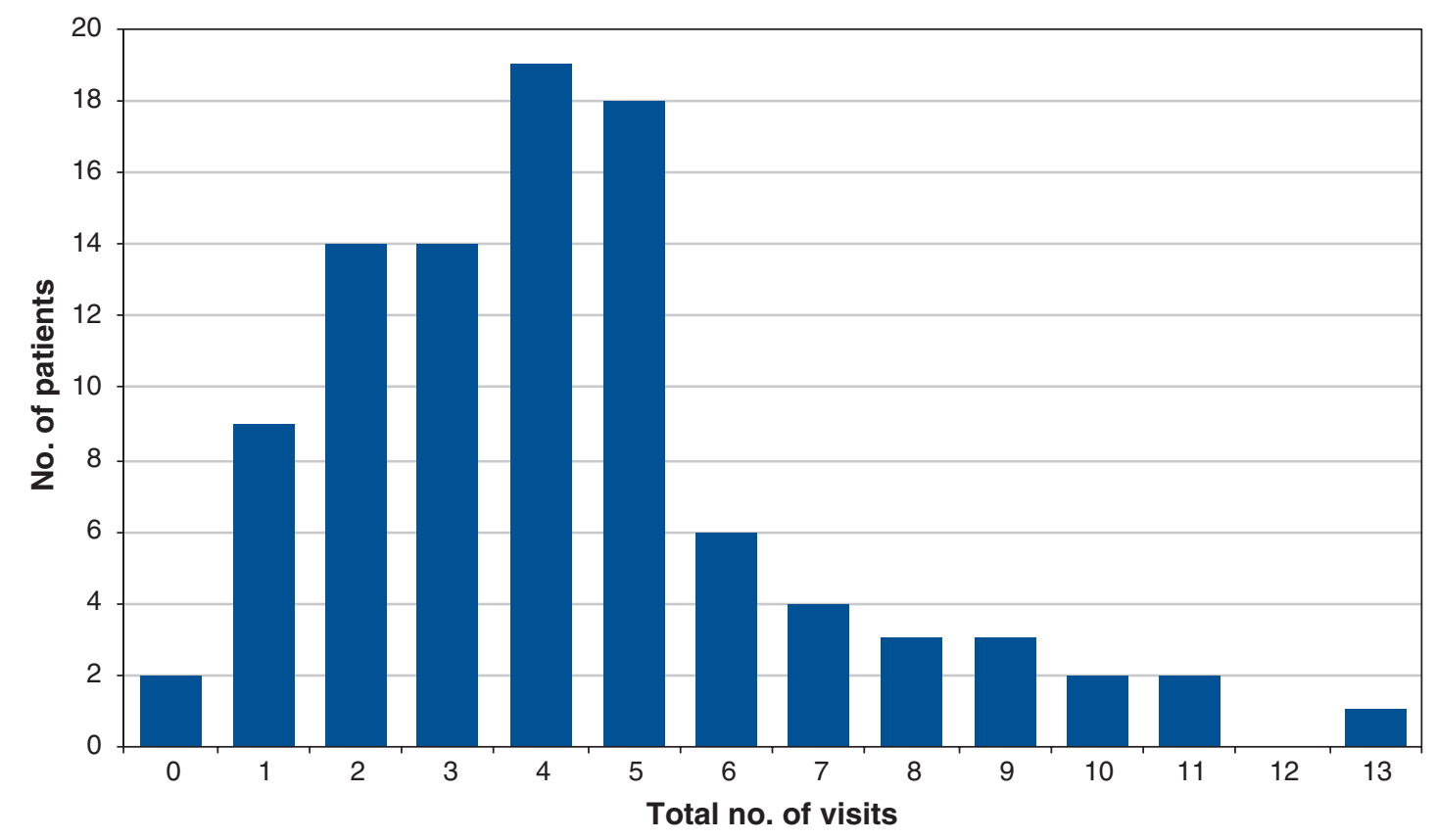

Figure 1: Histogram of total number of visits per patient. 


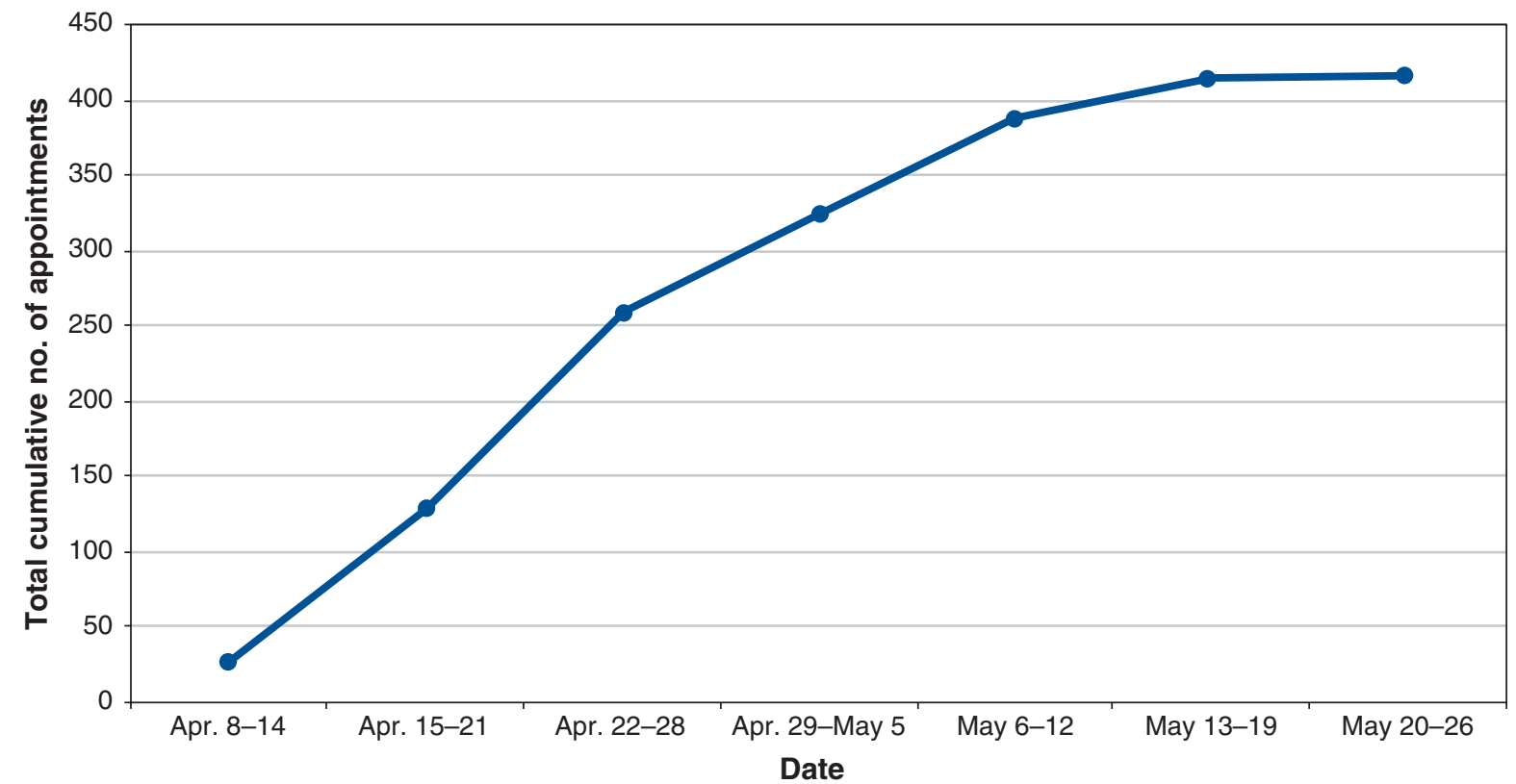

Figure 2: Line graph of cumulative number of appointments by week.

direct comparison is challenging owing to heterogenous patient populations and different data-reporting approaches.

Our model was family medicine led, whereas other programs were led by specialists or a mix of different clinicians. , $^{5,6,13,20}$ Initial results suggest our approach can manage the wide range of medical symptoms and comorbidities prevalent in patients

\begin{tabular}{|lc|}
\hline $\begin{array}{l}\text { Table 3: COVIDCare @ Home measures of program } \\
\text { feasibility }\end{array}$ & $\begin{array}{c}\text { No. (\%) of } \\
\text { patients } \\
n=97\end{array}$ \\
\hline Measure & $24(25)$ \\
\hline Patient sent a(n) & $5(5)$ \\
\hline Oximeter & $4(4)$ \\
\hline Thermometer & $2(2)$ \\
\hline $\begin{array}{l}\text { Virtual consultation with general internal } \\
\text { medicine physician, for the following reasons: }\end{array}$ & $1(1)$ \\
\hline Worsening symptoms & $1(1)$ \\
\hline Comorbidity management & $16(16)$ \\
\hline Rule out other disease & $9(9)$ \\
\hline $\begin{array}{l}\text { Virtual consultation with social worker, for the } \\
\text { following reasons: }\end{array}$ & $6(6)$ \\
\hline For support to find a primary care provider & $4(4)$ \\
\hline For support with financial or food insecurity & $6(6)$ \\
\hline For mental health support & $1(1)$ \\
\hline Pharmacy consultation & \\
\hline Acute ambulatory care unit consultation & \\
\hline
\end{tabular}

with COVID-19. Additional support by a specialist was required in a few cases and often could be provided virtually. Although $77 \%$ of patients reported having a primary care provider, many stayed in the program as it filled a gap in health care services during the early lockdown stage of the pandemic when access to primary care was reduced. The second wave of COVID-19 in Canada had a higher proportion of low-risk patients, so there is an opportunity to scale up this service by supporting community-based primary care providers to care for their own patients where possible.

Our team-based model and primary care expertise enabled us to support the mental health and social needs of patients, when appropriate. This was particularly relevant as $25 \%$ of our patients belonged to occupational groups (personal support, shelter and environmental services workers) that make them more likely to contract SARS-CoV-2 and more likely to have other risks for poor health outcomes. ${ }^{21}$ For example,

\begin{tabular}{|lc|}
\hline $\begin{array}{l}\text { Table 4: CoVIDCare@Home measures of } \\
\text { program safety }\end{array}$ & $\begin{array}{c}\text { No. }(\%) \text { of } \\
\text { patients } \\
n=97\end{array}$ \\
\hline Measure & $4(4)$ \\
\hline Emergency department visits & $2(2)$ \\
\hline Self-referred & $2(2)$ \\
\hline Referred through program & 0 \\
\hline Hospitalizations & 0 \\
\hline Deaths & \\
\hline
\end{tabular}


personal support workers are likely to be racialized, to be women and to have precarious employment arrangements. ${ }^{22}$ This aligns with several studies showing that women, people of colour and recent immigrants have higher rates of COVID19 and worse outcomes. ${ }^{23-26} \mathrm{~A}$ quarter of our patients did not have a regular primary care provider, which may reflect barriers including language, lack of access to local family physicians and difficulty navigating the health care system..$^{27,28}$

Although the digital divide remains a concern, the COVIDCare@Home model suggests that well-designed virtual health services may improve access to those who are typically underserved. For example, patients were able to access social workers or mental health providers at no cost, sometimes for critical resources such as access to food. Most published remote monitoring programs for COVID-19 did not include resources to support mental health or address the social determinants of health, though some planned to do so (1 reference from preprint). ${ }^{5,19}$ These are a key part of the care of people with COVID-19, to support them in maintaining quarantine and to ensure better outcomes.

\section{Limitations}

Given the early stage of this program, this study has several limitations. We have included the first 97 patients, who were enrolled in the first 5 weeks of the program. Further, the sample population was biased by the local COVID-19 testing prioritization at the time (focusing on health care workers). ${ }^{1}$ Patients who lived in long-term care settings were not included in the program, as there were other services available to support their unique health needs. Flexible use of telephones or video enabled broad access, but patients without access to a telephone could not participate. The initial model was fairly resource intensive, with coverage 7 days a week from all team members. The program was modified in response to fluctuations in need, and with the current rise in cases, the use of electronic surveys, remote monitoring apps, automated dashboards and greater integration of a primary care provider are being explored to rapidly adjust capacity. This study did not include a control group to measure efficacy directly, but a detailed programmatic evaluation including incorporation of patient and provider experience is underway to quantify the impact of COVIDCare@Home.

\section{Conclusion}

This study showed that a multidisciplinary, family medicineled remote monitoring program for outpatients with COVID19 is safe and feasible. The primary care model may be more adaptable to evolving patient and system needs, and easier to replicate in settings with limited access to specialty care. Given that certain populations are disproportionally affected by COVID-19, remote monitoring programs should consider how to improve health equity through increased virtual supports to address social determinants of health. Virtual care approaches like COVIDCare@Home that provide primary care support to patients, particularly those with more complex health and social needs, may be an important part of ongoing health system efforts to manage subsequent waves of COVID-19. Such approaches may also offer models for exploring holistic remote home monitoring subsequent to the pandemic.

\section{References}

1. Hospitalizations, intensive care unit (ICU), mechanical ventilation and deaths. Ottawa: Public Health Agency of Canada; updated 2021 Feb. 16. Available: https://health-infobase.canada.ca/covid-19/epidemiological-summary-covid-19 -cases.html\#a7 (accessed 2020 Dec. 13).

2. Greenhalgh T, Koh GCH, Car J. COVID-19: a remote assessment in primary care. BMF 2020;368:m1182.

3. Berlin DA, Gulick RM, Martinez FJ. Severe COVID-19. $N$ Engl 7 Med 2020;383:2451-60.

4. Gandhi RT, Lynch JB, Del Rio C. Mild or moderate COVID-19. N Engl f Med 2020;383:1757-66.

5. Medina M, Babiuch C, Card M, et al. Home monitoring for COVID-19. Cleve Clin 7 Med 2020 June 11 [Epub ahead of print]. doi: 10.3949/ccjm.87a. ccc028.

6. Lam PW, Sehgal P, Andany N, et al. A virtual care program for outpatients diagnosed with COVID-19: a feasibility study. CMAf Open 2020;8:E407-13.

7. Argenziano MG, Bruce SL, Slater CL, et al. Characterization and clinical course of 1000 patients with coronavirus disease 2019 in New York: retrospective case series. BM7 2020;369:m1996.

8. Song J-Y, Yun J-G, Noh J-Y, et al. COVID-19 in South Korea: challenges of subclinical manifestations. N Engl f Med 2020;382:1858-9.

9. Noh JY, Yoon JG, Seong H, et al. Asymptomatic infection and atypical manifestations of COVID-19: comparison of viral shedding duration. 7 Infect 2020;81:816-46

10. Naderpour Z, Saeedi M. A primer on COVID-19 for clinicians: clinical manifestation and natural course. Front Emerg Med 2020;4:e62.

11. Majeed A, Maile EJ, Bindman AB. The primary care response to COVID-19 in England's National Health Service. 7 R Soc Med 2020;113:208-10.

12. Williams S, Tsiligianni I. COVID-19 poses novel challenges for global primary care. NP7 Prim Care Respir Med 2020;30:30.

13. Kricke G, Roemer PE, Barnard C, et al. Rapid implementation of an outpatient COVID-19 monitoring program. NEFM Catal 2020 June 16. doi: 10.1056/CAT.20.0214.

14. Annis T, Pleasants S, Hultman G, et al. Rapid implementation of a COVID19 remote patient monitoring program. 7 Am Med Inform Assoc 2020;27: 1326-30.

15. McIsaac WJ, Upshur R, Kukan S. Challenges in the virtual assessment of COVID-19 infections in the community. Can Fam Physician 2021;67:e6-8.

16. Interim clinical guidance for management of patients with confirmed coronavirus disease (COVID-19). Atlanta: Centers for Disease Control and Prevention; 2020. Available: https://stacks.cdc.gov/view/cdc/89980 (accessed 2020 Nov. 20).

17. Case definition: coronavirus disease (COVID-19). Toronto: Ontario Ministry of Health; 2020. Available: www.health.gov.on.ca/en/pro/programs/public health/coronavirus/docs/2019_case_definition.pdf (accessed 2020 Nov. 20).

18. Proctor E, Silmere H, Raghavan R, et al. Outcomes for implementation research: conceptual distinctions, measurement challenges, and research agenda. Adm Policy Ment Health 2011;38:65-76.

19. Hutchings O, Dearing C, Jagers D, et al. Virtual health care for community management of patients with COVID-19 [preprint]. medRxiv 2020 May 15. doi: 10.1101/2020.05.11.20082396.

20. Xu H, Huang S, Qiu C, et al. Monitoring and management of home-quarantined patients with COVID-19 using a WeChat-based telemedicine system: retrospective cohort study. 7 Med Internet Res 2020;22:e19514.

21. COVID-19 and the social determinants of health: What do we know? Toronto: Toronto Public Health; 2020. Available: www.toronto.ca/wp-content/ uploads/2020/07/956b-SDOHandCOVID19_Summary_2020July1.pdf (accessed 2020 Nov. 20).

22. Van Houtven CH, DePasquale N, Coe NB. Essential long-term care workers commonly hold second jobs and double- or triple-duty caregiving roles. $7 \mathrm{Am}$ Geriatr Soc 2020;68:1657-60.

23. Laurencin CT, McClinton A. The COVID-19 pandemic: a call to action to identify and address racial and ethnic disparities. $\mathcal{F}$ Racial Ethn Health Disparities 2020;7:398-402.

24. Kantamneni N. The impact of the COVID-19 pandemic on marginalized populations in the United States: a research agenda. 7 Vocat Behav 2020; 119:103439.

25. COVID-19 in racial and ethnic minority groups. Atlanta: Centers for Disease Control and Prevention; updated 2020 Dec. 10. Available: www.cdc.gov/ coronavirus/2019-ncov/community/health-equity/racial-ethnic-disparities/ index.html (accessed 2020 Nov. 20).

26. Garg S, Kim L, Whitaker M, et al. Hospitalization rates and characteristics of patients hospitalized with laboratory-confirmed coronavirus disease 2019: COVID-NET, 14 states, March 1-30, 2020. MMWR Morb Mortal Wkly Rep 2020;69:458-64. 
27. Glazier RH, Gozdyra P, Kim M, et al. Geographic variation in primary care need, service use and providers in Ontario, 2015/16. Toronto: ICES; 2018.

28. Ahmed S, Shommu NS, Rumana N, et al. Barriers to access of primary healthcare by immigrant populations in Canada: a literature review. $\mathcal{F}$ Immigr Minor Health 2016;18:1522-40.

Affiliations: Women's College Hospital Institute for Health System Solutions and Virtual Care (Agarwal, Mukerji, Laur, Chandra, Bhatia, Bhattacharyya), and Department of Family and Community Medicine (Agarwal, Bhattacharyya, Martin), and Division of Endocrinology \& Metabolism (Mukerji), Department of Medicine, and Women's College Hospital Academic Family Health Team (Pimlott, Heisey), Department of Family and Community Medicine, and Division of General Internal Medicine (Stovel), Department of Medicine, University of Toronto; Division of General Internal Medicine (Stovel), Women's College Hospital; Women's College Hospital (Goulbourne, Martin); University Health Network (Bhatia); Dalla Lana School of Public Health (Martin), University of Toronto, Toronto, Ont.

Contributors: Payal Agarwal led on all aspects of this program and manuscript. Geetha Mukerji supported the conception of the study and supervised the overall design and activities of the manuscript. Celia Laur contributed to the evaluation of the program and the comparison to other literature. Shivani Chandra contributed to the evaluation of the program and supported the data analysis. Nick Pimlott, Ruth Heisey, Rebecca Stovel and Elaine Goulbourne supported the conception and development of the program and development of the manuscript. R. Sacha Bhatia, Onil Bhattacharyya and Danielle Martin provide oversight to the program development and delivery, and supported manuscript conceptualization and writing. Payal Agarwal drafted the manuscript. All of the authors revised the manuscript, gave final approval of the version to be published and agreed to be accountable for all aspects of the work.

Funding: There was no direct funding provided for this study. Payal Agarwal is funded in part by a New Investigator Award from the Department of Family and Community Medicine at the University of Toronto. Celia Laur is funded by the Canadian Institutes of Health Research, Health System Impact Fellowship (postdoctoral). This study was completed by the investigators without the influence of any commercial sponsor.

Content licence: This is an Open Access article distributed in accordance with the terms of the Creative Commons Attribution (CC BY-NC-ND 4.0) licence, which permits use, distribution and reproduction in any medium, provided that the original publication is properly cited, the use is noncommercial (i.e., research or educational use), and no modifications or adaptations are made. See: https://creativecommons.org/licenses/ by-nc-nd/4.0/

Data sharing: Portions of the data are available on request to the corresponding author.

Acknowledgements: The authors thank the full team of clinicians and partners who developed and continue to deliver the COVIDCare@Home program. Further thanks to the patients who participated in this program.

Supplemental information: For reviewer comments and the original submission of this manuscript, please see www.cmajopen.ca/content/9/2/ E324/suppl/DC1. 